Supporting Information

\title{
Poly(ionic liquid)-Stabilized Graphene Nanoinks for Scalable 3D Printing of Graphene Aerogels
}

\author{
Tuan Sang Tran, Naba Kumar Dutta, Namita Roy Choudhury* \\ Chemical and Environmental Engineering, School of Engineering, RMIT University \\ Melbourne, Victoria 3000, Australia. \\ "Corresponding author. Email: namita.choudhury@ rmit.edu.au.
}

\section{Table of Contents}

1. Experimental Section

1.1. Preparation of electrochemically exfoliated graphite

1.2. Synthesis of immidazolium-based poly(ionic liquid)s

1.3. Optimization of the parameters for 3D printing

1.4. Characterization

2. Tuning the solubility of poly(ionic liquid)s for graphite exfoliation in low boiling point solvents

Scheme S1: Anion-exchange of imidazolium-based poly(ionic liquid)s.

Figure S1: Comparison of graphite exfoliation in acetone, with and without poly(ionic liquid)s.

3. The temperature dependence of ink viscosity

Figure S2: Shear-rate-dependent viscosity profiles of the inks at different temperature.

4. Calculation of printing indicators

Table S1. Printing indicator of the concentrated graphene ink stabilized by poly(ionic liquid)s

5. Electrical conductivity measurement

Figure S3: Four-point probe setup for electrical conductivity measurement.

6. Electrochemical performance of the thin-film electrodes.

Figure S4: Electrochemical performance of the graphene/poly(ionic liquid)s thin-films (stacked electrodes).

7. The fabricated supercapacitor device

Figure S5: Photograph of the fabricated supercapacitor device. 


\section{Experimental Section}

\subsection{Preparation of electrochemically exfoliated graphite}

The electrochemical exfoliation of graphite was carried out in a two-electrode system using anodic approach, where a graphite electrode was used as the anode and a platinum electrode was used as the cathode. High purity graphite rods $(99.995 \%$ trace metals basis, $3 \mathrm{~mm}$ in diameter and $150 \mathrm{~mm}$ in length) were pre-treated by alternately soaked in liquid nitrogen and absolute ethanol, and dried in oven at $70{ }^{\circ} \mathrm{C}$ for $24 \mathrm{~h}$. After dried, a graphite rod was placed parallel to a platinum electrode with a fixed distance of $4 \mathrm{~cm}$ and connected to the power source. As electrolyte, $200 \mathrm{mg}$ of 2,2,6,6tetramethyl-1-piperidinyloxy (TEMPO) was dissolved in $200 \mathrm{~mL}$ of $0.05 \mathrm{M}$ ammonium sulfate $\left[\left(\mathrm{NH}_{4}\right)_{2} \mathrm{SO}_{4}\right]$ aqueous solution. Both electrodes were immersed in the electrolyte with $10 \mathrm{~cm}$ effective length exposed to the solution. A positive voltage of $10 \mathrm{~V}$ was applied to the graphite anode to start the electrochemical exfoliation process using an Instek GPR-6030D power supply. During this process, gas bubbles were formed in both electrodes, with the graphite anode gradually expanded and released graphitic fragments from its surface. When the exfoliation was completed, the product was filtered and washed alternatively with water and ethanol. The final solid materials were dried under vacuum overnight, resulted in electrochemically exfoliated graphite with increased space between graphitic layers.

\subsection{Synthesis of immidazolium-based poly(ionic liquid)s}

The monomer, 1-vinyl-3-ethyl-ethylimidazolium bromide (ViEtImBr) was firstly prepared by refluxing 1-vinylimidazole $(10 \mathrm{~g})$ and bromoethane $(20 \mathrm{~g})$ at $70{ }^{\circ} \mathrm{C}$ for $24 \mathrm{~h}$. The resultant white solid was washed several times with ethyl acetate and dried in a vacuum oven for 2 days (yield: 66\%, 14.25 g). Afterward, polymerization was carried out in chloroform with azobisisobutyronitrile (AIBN) radical initiator. $3 \mathrm{~g}$ of the as-synthesized monomer (ViEtImBr) was dissolved in $30 \mathrm{~mL}$ of chloroform in a round-bottom flask and heated at $70{ }^{\circ} \mathrm{C}$ under $\mathrm{N}_{2}$ atmosphere. Then, $0.06 \mathrm{~g}$ of AIBN was dissolved in $3 \mathrm{~mL}$ of chloroform and slowly injected into the reaction vessel. The mixture was 
refluxed for $3 \mathrm{~h}$, resulted in poly $(\mathrm{ViEtImBr})$ as a white solid that was washed several times with chloroform and dried under vacuum overnight (yield: 97\%, $2.92 \mathrm{~g}$ ).

Similarly, 1-vinyl-3-butylimidazolium chloride ( $\mathrm{ViBuImCl})$ was prepared by direct reaction of 1vinylimidazole (10 g) with 1-chlorobutane $(20 \mathrm{~g})$ at $70^{\circ} \mathrm{C}$ for $48 \mathrm{~h}$. Phase separation occurred and the monomer was obtained as an amber viscous liquid that was precipitated in ethyl acetate under vigorous shaking. After washing several times with ethyl acetate, ViBuImCl was obtained as a whiteyellow solid which was dried in a vacuum oven for 2 days prior to polymerization (yield: $44 \%, 8.65$ g). The polymerization of $\mathrm{ViBuImCl}$ was similar to the previous described procedures for $\mathrm{ViEt} \operatorname{ImBr}$ except for the reaction time increased to $24 \mathrm{~h}$. After cooling to room temperature, the mixture was precipitated with the addition of diethyl ether. The final product, poly(ViBuImCl), was obtained as a white solid after washed several times with diethyl ether and dried in a vacuum oven for $24 \mathrm{~h}$ (yield: $95 \%, 2.85 \mathrm{~g})$.

\subsection{Optimization of the parameters for $3 D$ printing}

The 3D printer used in this work (GeSiM BioScaffolder 3.1) allows for the use of multiple printing nozzles simultaneously with versatile printing conditions. As the temperature plays an important role on the rheology of the inks, the syringe holder was equipped with a temperature controller and kept at $25^{\circ} \mathrm{C}$ during operation. We used a wide range of nozzles with size varying from $20 \mathrm{GA}$ to $34 \mathrm{GA}$ (length: $25.4 \mathrm{~mm}$, their inner diameters are shown in Table. S1). It is noted that the formulated graphene inks were ejectable through all printing nozzles. However, we mostly used the 25 GA nozzle as it could deliver high resolution while maintaining a reliable printing performance without any blockage. The step height between two layers was $0.5 \mathrm{~mm}$. The offset gap between the nozzle and the substrate was set at $0.5 \mathrm{~mm}$ to prevent collision between the printing head and the cryogenized printed patterns during printing. The extrusion rate was controlled by the applied pressure and was set as $20 \mathrm{kPa}$, which was found to be optimal for the printing speed of $10 \mathrm{~mm} \mathrm{~s}^{-1}$. 


\subsection{Characterization}

Proton nuclear magnetic resonance $\left({ }^{1} \mathrm{H}\right.$ NMR) analysis was carried out on a Bruker AVANCE $300 \mathrm{MHz}$ spectrometer using deuterium oxide $\left(\mathrm{D}_{2} \mathrm{O}\right)$ as the solvent. Fourier-transform infrared spectroscopic (FT-IR) scans were acquired using a PerkinElmer Spectrum 100 spectrometer. Surface tension of the dispersions were measured using a Kruss DSA25 tensiometer. Shear viscosity measurements were performed on a TA Instruments HR-3 Discovery hybrid rheometer. Scanning electron microscopy (SEM) was carried out on a FEI Nova NanoSEM 200. Transmission electron microscopy (TEM) was carried out on a JEOL 1010 TEM. UV-visible spectroscopy was performed using a Shimadzu UV-2600. Raman spectrum was recorded using a HORIBA LabRAM HR Evolution with a $532 \mathrm{~nm}$ laser excitation. X-ray photoelectron spectroscopy (XPS) measurement was performed using a Thermo Scientific K-Alpha with a monochromated Al K $\alpha$ X-ray source. The sheet resistance was measured by a four-point probe using a Keithley 2450 source meter. Cyclic voltammetry (CV) and cyclic charge/discharge (CCD) experiments were carried out using a Gamry Interface 1010E Potentiostat. 


\section{Tuning the solubility of poly(ionic liquid)s for graphite exfoliation in low boiling point solvents}

It has been established that the solubility of poly(ionic liquid)s can be tuned by employing different counteranions in its polymeric backbone. Thus, we utilized a simple anion-exchange process to produce acetone-soluble imidazolium-based poly(ionic liquid)s from its water-soluble counterparts. Water-soluble poly(ionic liquid)s was dissolved in deionized water (1 g, $20 \mathrm{~mL})$ and mixed with an excess of Lithium bis(trifluoromethylsulfonyl)amide aqueous solution (6 g, $12 \mathrm{~mL})$. The mixtures were added to a 50-mL, round-bottom flask and stirred vigorously for $1 \mathrm{~h}$ at room temperature. Immediately after mixing, new polymers precipitated quantitatively as a result of the anion-exchange reaction. The precipitations were filtered, washed several times, and dried in a vacuum oven until constant weight. The anion-exchange process is shown in scheme S1.

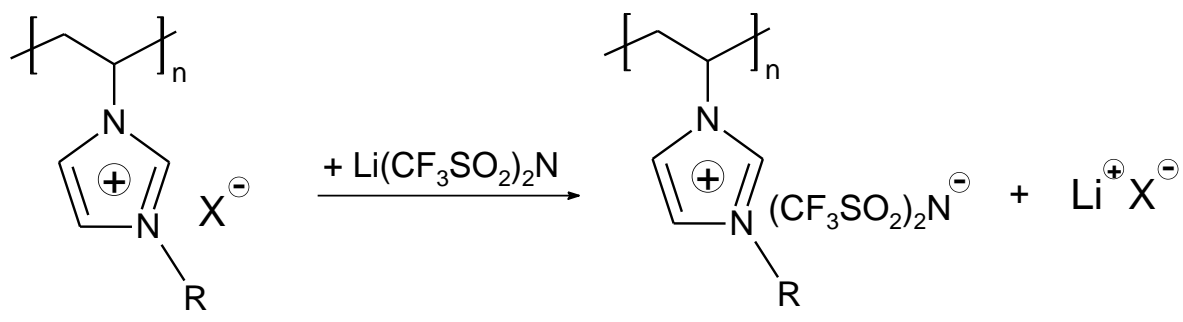

Scheme S1: Anion-exchange of imidazolium-based poly(ionic liquid)s.

The as-prepared acetone-soluble poly(ionic liquid)s were used as stabilizers for graphite exfoliation in acetone. Typically, $5 \mathrm{~g}$ of graphite and $1 \mathrm{~g}$ of poly(ionic liquid)s were mixed into $100 \mathrm{~mL}$ of acetone. The mixtures were then placed in an ice bath and sonicated for $60 \mathrm{~min}$. After sonication, the dispersions were mild-centrifuged at 2,000 rpm for $30 \mathrm{~min}$ to remove any unexfoliated graphite particles. For comparison, exfoliation of $5 \mathrm{~g}$ of graphite in $100 \mathrm{~mL}$ of acetone without the presence of poly(ionic liquid)s were also performed with similar processing parameters. While graphene dispersions in acetone were unstable and aggregated in less than a day, graphene dispersions stabilized by poly(ionic liquid)s were stable for over a week without notable precipitation. The comparison is shown in Fig. S1. 


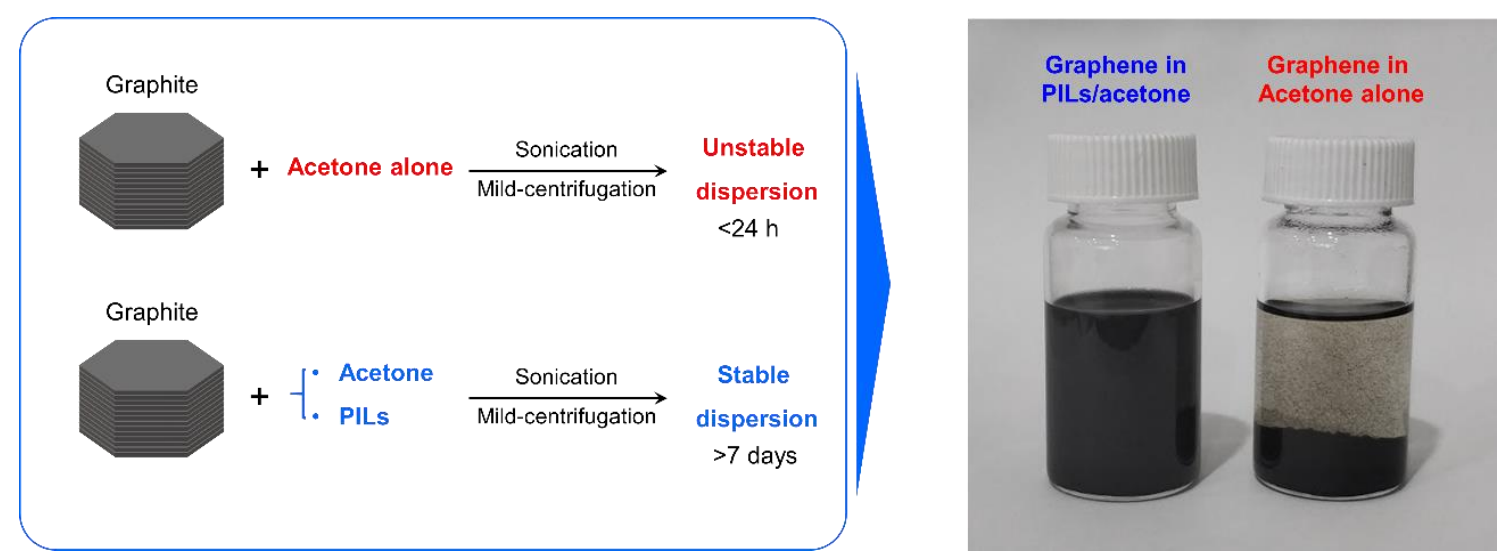

Figure S1: Comparison of graphite exfoliation in acetone, with and without poly(ionic liquid)s. 


\section{The temperature dependence of ink viscosity}

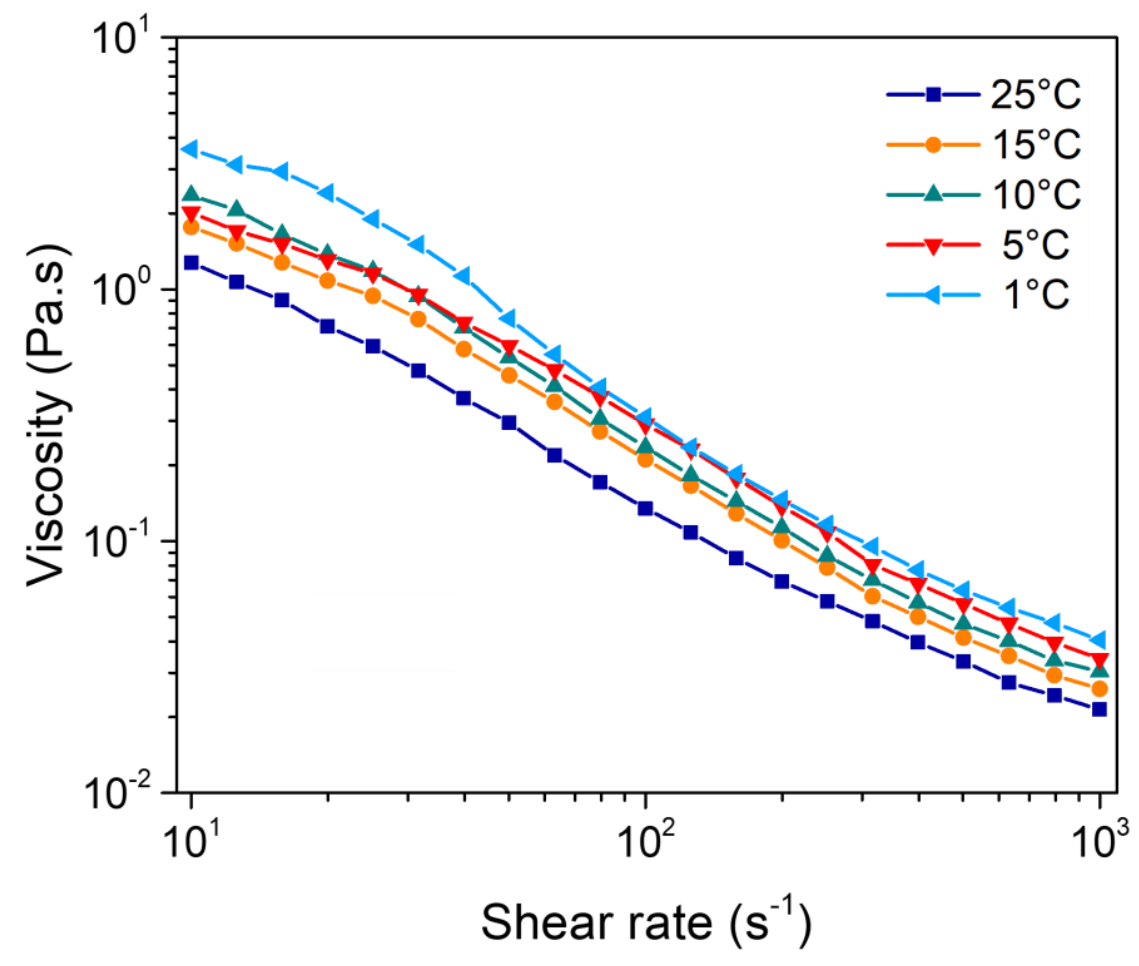

Figure S2: Shear-rate-dependent viscosity profiles of the Poly(ViEtImBr)-based inks at different temperature 


\section{Calculation of printing indicators}

Table S1. Printing indicator of the concentrated graphene ink stabilized by poly(ionic liquid)s with various nozzle sizes at different shear rates.

Graphene ink [5 mg mL $\mathrm{m}^{-1}$ ] stabilized by poly(ViEtImBr) [10 $\mathrm{mg} \mathrm{mL}^{-1}$ ]

\begin{tabular}{|c|c|c|c|c|c|c|}
\hline Nozzle size & $\begin{array}{c}\text { Shear rate } \\
\left(\mathrm{s}^{-1}\right)\end{array}$ & $\begin{array}{l}\text { Viscosity } \\
\text { (Pa.s) }\end{array}$ & $\begin{array}{l}\text { Density } \\
\left(\mathrm{kg} \mathrm{m}^{-3}\right)\end{array}$ & $\begin{array}{c}\text { Inner diameter } \\
(\mathrm{mm})\end{array}$ & $\begin{array}{l}\text { Surface tension } \\
\qquad\left(\mathrm{mN} \mathrm{m}^{-1}\right)\end{array}$ & Printing indicator \\
\hline \multirow{7}{*}{$34 \mathrm{GA}$} & 10 & 1.28 & \multirow{7}{*}{1000} & \multirow{7}{*}{0.08} & \multirow{7}{*}{76} & 0.06 \\
\hline & 25 & 0.59 & & & & 0.13 \\
\hline & 50 & 0.30 & & & & 0.26 \\
\hline & 100 & 0.16 & & & & 0.58 \\
\hline & 250 & 0.06 & & & & 1.35 \\
\hline & 500 & 0.03 & & & & 2.34 \\
\hline & 1000 & 0.02 & & & & 3.63 \\
\hline \multirow{7}{*}{ 30GA } & 10 & 1.28 & \multirow{7}{*}{1000} & \multirow{7}{*}{0.16} & \multirow{7}{*}{76} & 0.09 \\
\hline & 25 & 0.59 & & & & 0.19 \\
\hline & 50 & 0.30 & & & & 0.37 \\
\hline & 100 & 0.16 & & & & 0.82 \\
\hline & 250 & 0.06 & & & & 1.91 \\
\hline & 500 & 0.03 & & & & 3.30 \\
\hline & 1000 & 0.02 & & & & 5.13 \\
\hline \multirow{7}{*}{$27 \mathrm{GA}$} & 10 & 1.28 & \multirow{7}{*}{1000} & \multirow{7}{*}{0.21} & \multirow{7}{*}{76} & 0.10 \\
\hline & 25 & 0.59 & & & & 0.21 \\
\hline & 50 & 0.30 & & & & 0.43 \\
\hline & 100 & 0.16 & & & & 0.94 \\
\hline & 250 & 0.06 & & & & 2.19 \\
\hline & 500 & 0.03 & & & & 3.78 \\
\hline & 1000 & 0.02 & & & & 5.88 \\
\hline \multirow{7}{*}{$25 \mathrm{GA}$} & 10 & 1.28 & \multirow{7}{*}{1000} & \multirow{7}{*}{0.26} & \multirow{7}{*}{76} & 0.11 \\
\hline & 25 & 0.59 & & & & 0.24 \\
\hline & 50 & 0.30 & & & & 0.48 \\
\hline & 100 & 0.16 & & & & 1.04 \\
\hline & 250 & 0.06 & & & & 2.43 \\
\hline & 500 & 0.03 & & & & 4.21 \\
\hline & 1000 & 0.02 & & & & 6.55 \\
\hline \multirow{7}{*}{ 20GA } & 10 & 1.28 & \multirow{7}{*}{1000} & \multirow{7}{*}{0.6} & \multirow{7}{*}{76} & 0.17 \\
\hline & 25 & 0.59 & & & & 0.36 \\
\hline & 50 & 0.30 & & & & 0.72 \\
\hline & 100 & 0.16 & & & & 1.58 \\
\hline & 250 & 0.06 & & & & 3.69 \\
\hline & 500 & 0.03 & & & & 6.40 \\
\hline & 1000 & 0.02 & & & & 9.94 \\
\hline \multirow{7}{*}{$15 \mathrm{GA}$} & 10 & 1.28 & & & & 0.25 \\
\hline & 25 & 0.59 & & & & 0.54 \\
\hline & 50 & 0.30 & & & & 1.09 \\
\hline & 100 & 0.16 & 1000 & 1.37 & 76 & 2.39 \\
\hline & 250 & 0.06 & & & & 5.58 \\
\hline & 500 & 0.03 & & & & 9.67 \\
\hline & 1000 & 0.02 & & & & 15.02 \\
\hline & 10 & 1.28 & & & & 0.40 \\
\hline & 25 & 0.59 & & & & 0.86 \\
\hline & 50 & 0.30 & & & & 1.73 \\
\hline 08GA & 100 & 0.16 & 1000 & 3.43 & 76 & 3.78 \\
\hline & 250 & 0.06 & & & & 8.83 \\
\hline & 500 & 0.03 & & & & 15.29 \\
\hline & 1000 & 0.02 & & & & 23.77 \\
\hline
\end{tabular}




\begin{tabular}{|c|c|c|c|c|c|c|}
\hline \multicolumn{7}{|c|}{ Graphene ink [5 $\left.\mathrm{mg} \mathrm{mL}^{-1}\right]$ stabilized by poly(ViBuImCl) [10 $\left.\mathrm{mg} \mathrm{mL}^{-1}\right]$} \\
\hline Nozzle size & $\begin{array}{c}\text { Shear rate } \\
\left(\mathrm{s}^{-1}\right)\end{array}$ & $\begin{array}{l}\text { Viscosity } \\
\text { (Pa.s) }\end{array}$ & $\begin{array}{l}\text { Density } \\
\left(\mathrm{kg} \mathrm{m}^{-3}\right)\end{array}$ & $\begin{array}{c}\text { Inner diameter } \\
(\mathrm{mm})\end{array}$ & $\begin{array}{l}\text { Surface tension } \\
\qquad\left(\mathrm{mN} \mathrm{m}^{-1}\right)\end{array}$ & Printing indicator \\
\hline \multirow{7}{*}{$34 \mathrm{GA}$} & 10 & 3.05 & \multirow{7}{*}{1000} & \multirow{7}{*}{0.08} & \multirow{7}{*}{$(2-1)}$, & 0.03 \\
\hline & 25 & 1.33 & & & & 0.06 \\
\hline & 50 & 0.53 & & & & 0.14 \\
\hline & 100 & 0.23 & & & & 0.34 \\
\hline & 250 & 0.09 & & & & 0.84 \\
\hline & 500 & 0.06 & & & & 1.35 \\
\hline & 1000 & 0.04 & & & & 2.05 \\
\hline \multirow{7}{*}{ 30GA } & 10 & 3.05 & \multirow{7}{*}{1000} & \multirow{7}{*}{0.16} & \multirow{7}{*}{73} & 0.04 \\
\hline & 25 & 1.33 & & & & 0.08 \\
\hline & 50 & 0.53 & & & & 0.20 \\
\hline & 100 & 0.23 & & & & 0.48 \\
\hline & 250 & 0.09 & & & & 1.19 \\
\hline & 500 & 0.06 & & & & 1.91 \\
\hline & 1000 & 0.04 & & & & 2.90 \\
\hline \multirow{7}{*}{$27 \mathrm{GA}$} & 10 & 3.05 & \multirow{7}{*}{1000} & \multirow{7}{*}{0.21} & \multirow{7}{*}{73} & 0.04 \\
\hline & 25 & 1.33 & & & & 0.09 \\
\hline & 50 & 0.53 & & & & 0.23 \\
\hline & 100 & 0.23 & & & & 0.55 \\
\hline & 250 & 0.09 & & & & 1.36 \\
\hline & 500 & 0.06 & & & & 2.19 \\
\hline & 1000 & 0.04 & & & & 3.32 \\
\hline \multirow{7}{*}{$25 \mathrm{GA}$} & 10 & 3.05 & \multirow{7}{*}{1000} & \multirow{7}{*}{0.26} & \multirow{7}{*}{73} & 0.05 \\
\hline & 25 & 1.33 & & & & 0.10 \\
\hline & 50 & 0.53 & & & & 0.26 \\
\hline & 100 & 0.23 & & & & 0.61 \\
\hline & 250 & 0.09 & & & & 1.51 \\
\hline & 500 & 0.06 & & & & 2.44 \\
\hline & 1000 & 0.04 & & & & 3.69 \\
\hline \multirow{7}{*}{$20 \mathrm{GA}$} & 10 & 3.05 & \multirow{7}{*}{1000} & \multirow{7}{*}{0.6} & & 0.07 \\
\hline & 25 & 1.33 & & & & 0.16 \\
\hline & 50 & 0.53 & & & & 0.39 \\
\hline & 100 & 0.23 & & & 73 & 0.93 \\
\hline & 250 & 0.09 & & & & 2.30 \\
\hline & 500 & 0.06 & & & & 3.71 \\
\hline & 1000 & 0.04 & & & & 5.61 \\
\hline & 10 & 3.05 & & & & 0.10 \\
\hline & 25 & 1.33 & & & & 0.24 \\
\hline & 50 & 0.53 & & & & 0.59 \\
\hline $15 \mathrm{GA}$ & 100 & 0.23 & 1000 & 1.37 & 73 & 1.42 \\
\hline & 250 & 0.09 & & & & 3.48 \\
\hline & 500 & 0.06 & & & & 5.60 \\
\hline & 1000 & 0.04 & & & & 8.48 \\
\hline & 10 & 3.05 & & & & 0.16 \\
\hline & 25 & 1.33 & & & & 0.38 \\
\hline & 50 & 0.53 & & & & 0.94 \\
\hline 08GA & 100 & 0.23 & 1000 & 3.43 & 73 & 2.23 \\
\hline & 250 & 0.09 & & & & 5.50 \\
\hline & 500 & 0.06 & & & & 8.86 \\
\hline & 1000 & 0.04 & & & & 13.42 \\
\hline
\end{tabular}




\section{Electrical conductivity measurement}

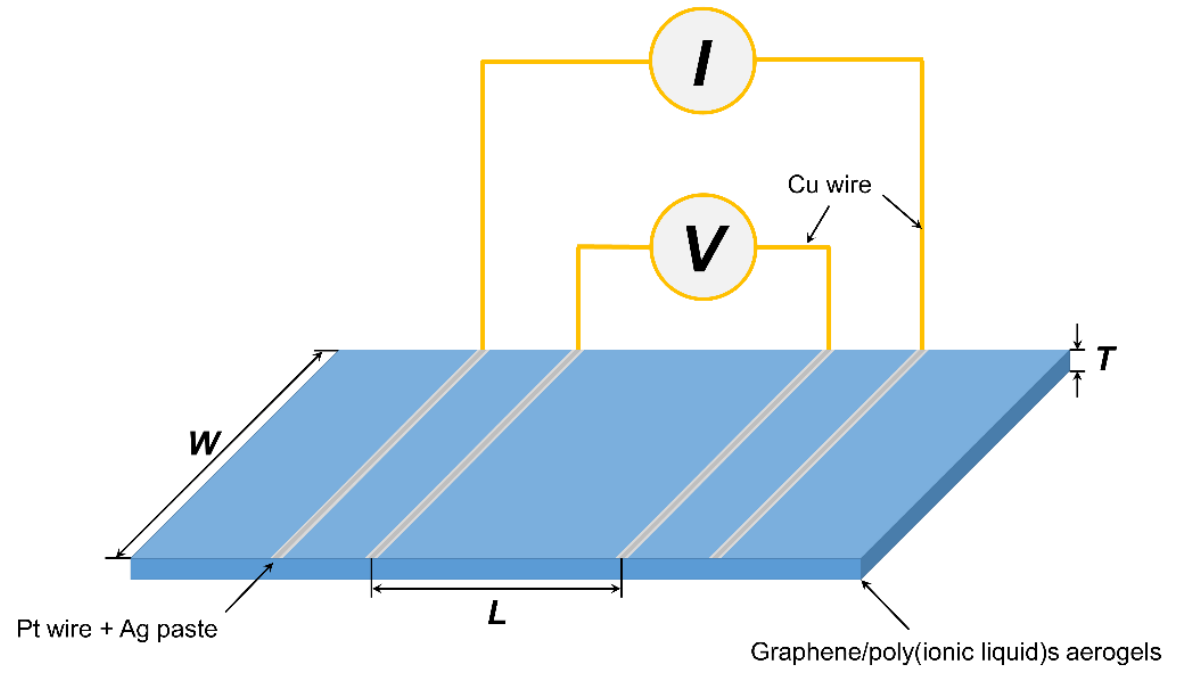

Figure S3: Four-point probe setup for electrical conductivity measurement.

The electrical conductivity of the 3D printed graphene/poly(ionic liquid)s aerogels was calculated according to the following formulae:

$$
\sigma=\frac{1}{\rho}=\frac{L}{R \times S}=\frac{I \times L}{V \times W \times T}
$$

Where: $\sigma$ and $\rho$ are the electrical conductivity and the electrical resistivity.

$L, R$, and $S$ are conductive length, cross-sectional area, and electrical resistance.

$I$ and $V$ are the measured current and voltage, respectively.

$W$ and $T$ are the width and the height of the aerogel. 
(a)

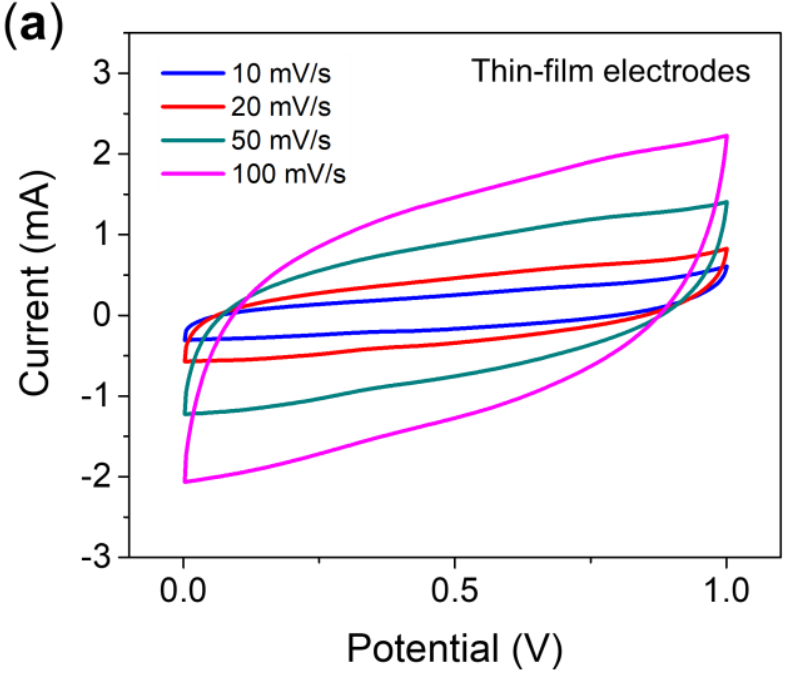

(c)

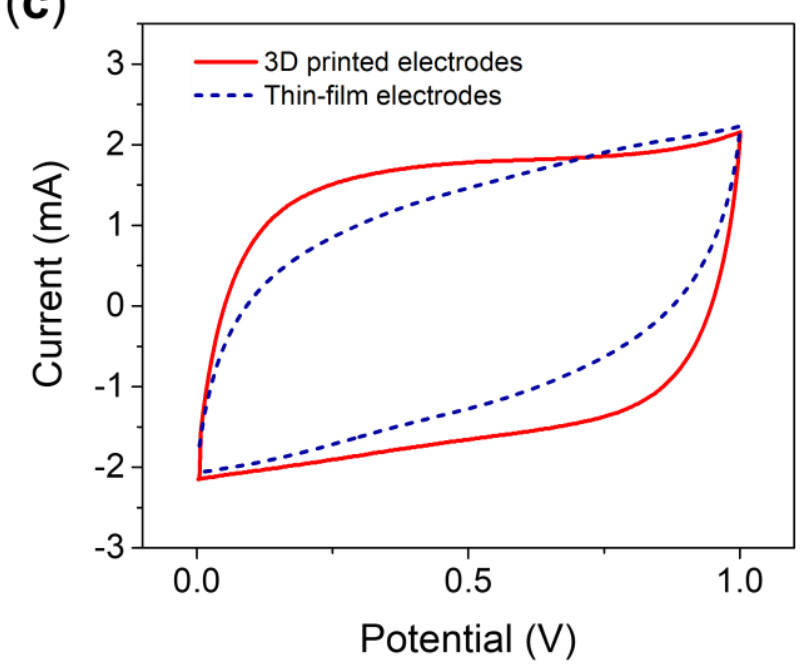

(b)

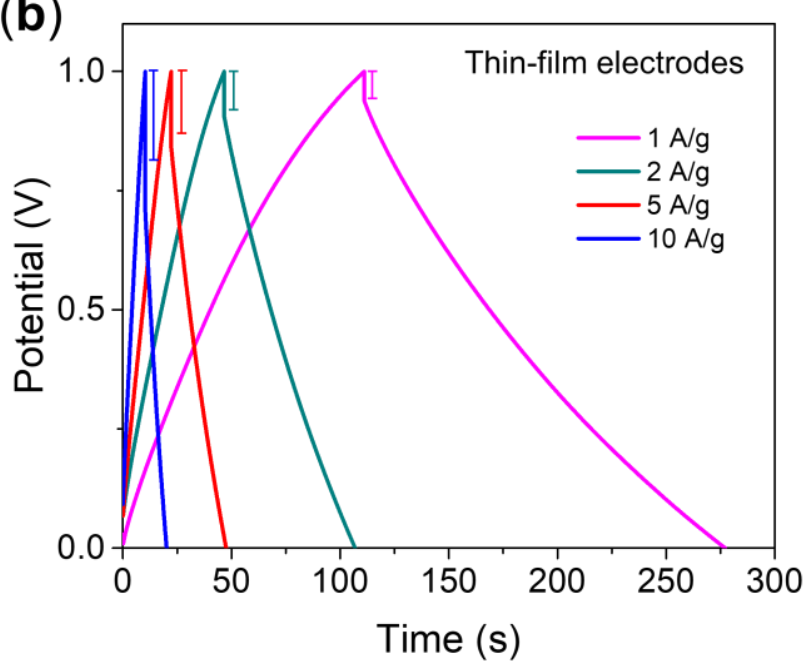

(d)

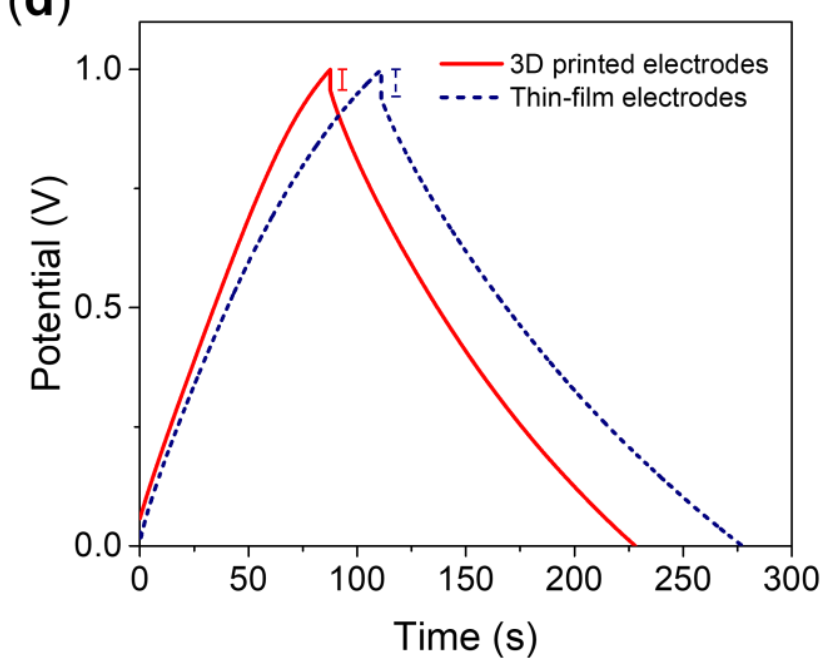

Figure S4: Electrochemical performance of the graphene/poly(ionic liquid)s thin-films (stacked electrodes). (a) CV curves of the thin-film electrodes at different scan rates. (b) CCD curves the thin-film electrodes at different current densities. (c) Comparison for the CV curves of the thin-film electrodes and the graphene/poly(ionic liquid)s aerogels (3D printed electrodes) at a scan rate of $100 \mathrm{mV} / \mathrm{s}$. (d) Comparison for the CCD curves of the thin-film electrodes and the 3D printed electrodes at a current density of $1 \mathrm{~A} / \mathrm{g}$. 


\section{The fabricated supercapacitor device.}
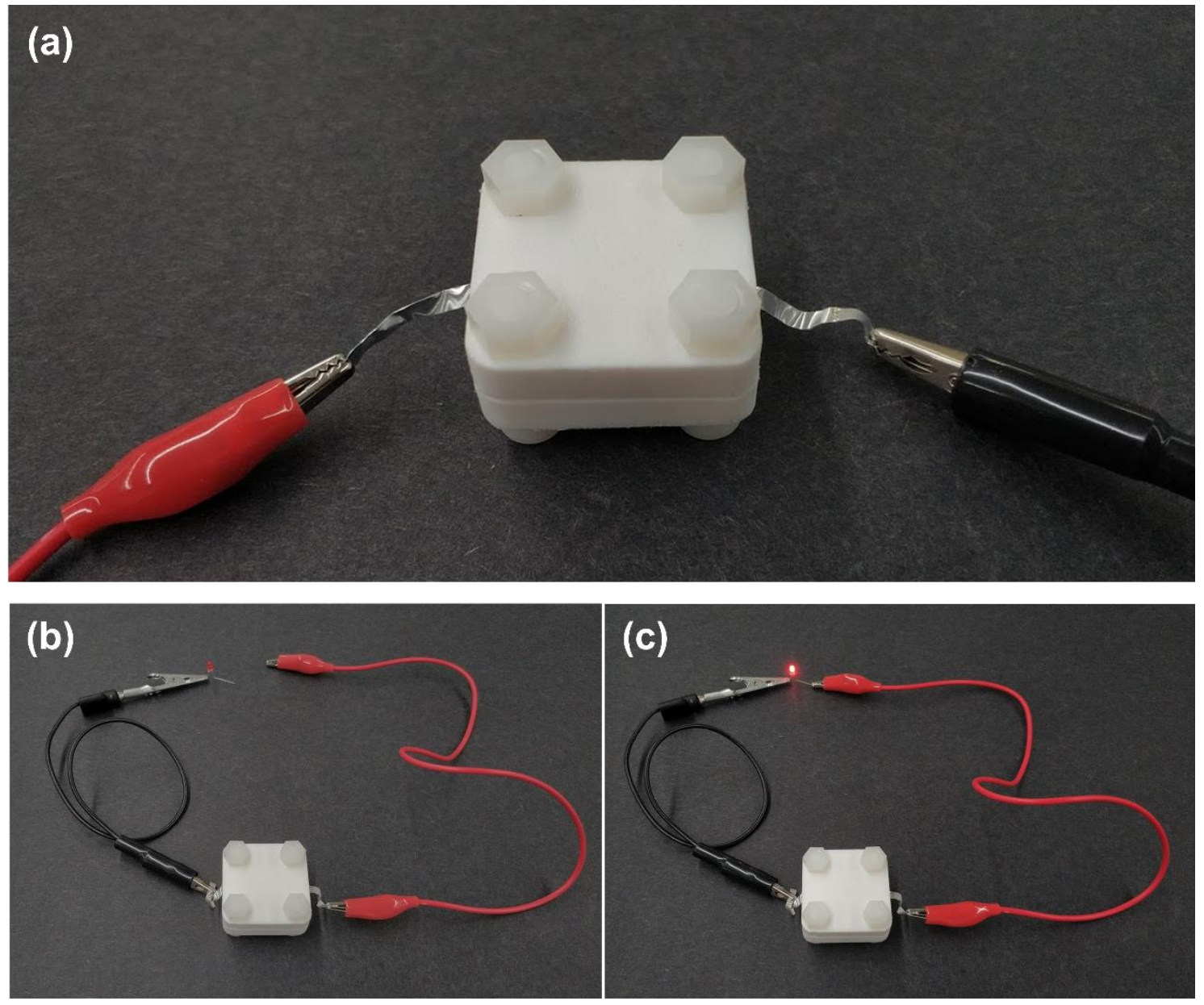

Figure S5: (a) Photograph of the fabricated supercapacitor device. (b, c) Photograph of a LED chip powered by the fabricated supercapacitor. 